\title{
Belphégor
}

\section{Andrew Burn and Chris Richards (eds) Children's Games in the New Media Age. Childlore, Media and Playground}

Gilles Brougère

\section{(2) OpenEdition}

Journals

Electronic version

URL: http://journals.openedition.org/belphegor/730

DOI: $10.4000 /$ belphegor.730

ISSN: 1499-7185

Publisher

LPCM

\section{Electronic reference}

Gilles Brougère, «Andrew Burn and Chris Richards (eds) Children's Games in the New Media Age. Childlore, Media and Playground », Belphégor [Online], 14 | 2016, Online since 10 October 2016,

connection on 10 December 2020. URL : http://journals.openedition.org/belphegor/730 ; DOI : https:// doi.org/10.4000/belphegor.730

This text was automatically generated on 10 December 2020.

\section{cc) (i) (2)}

Belphégor est mis à disposition selon les termes de la Licence Creative Commons Attribution - Pas d'Utilisation Commerciale - Pas de Modification 4.0 International. 


\section{Andrew Burn and Chris Richards (eds) Children's Games in the New Media Age. Childlore, Media and Playground}

Gilles Brougère

\section{REFERENCES}

Andrew Burn and Chris Richards (eds) Children's Games in the New Media Age.

Childlore, Media and Playground, Farhnam, Ashgate, 2014 
Children's Games in the

New Media Age

Childlore. Media and the Playground

Edited by ANDREW BURN and CHRIS RICHARDS

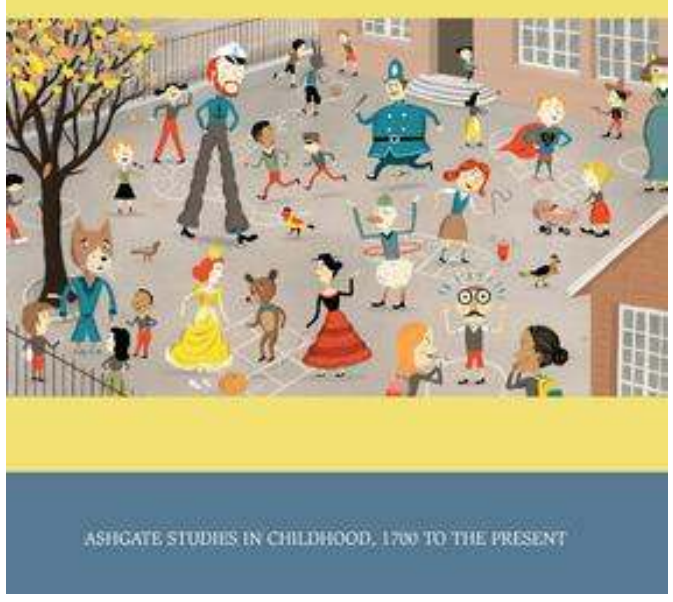

1 Cet ouvrage dirigé par deux professeurs de l'Institute of Education de l'Université de Londres rend compte d'une recherche collective impliquant plusieurs chercheurs de différentes institutions dont la British Library et articulant leurs réflexions autour des jeux de cour de récréation (playground) des enfants ${ }^{1}$.

2 Cette recherche se situe explicitement et volontairement dans la continuité de celles effectuées par les époux Opie, célèbre couple de chercheurs anglais ayant publié pendant trente ans plusieurs livres sur les chants et jeux de rue ou de cour de récréation en Angleterre ${ }^{2}$. Ils ont produit un travail de recueil traditionnel ou d'enregistrements audio, dans la logique des études folkloriques, en particulier dans une école où ils sont régulièrement revenus durant ces trente années. Cela est d'autant plus central qu'une dimension de la recherche liée à l'implication de la British Library avait pour objectif de numériser et de rendre accessible les recueils réalisés avec des systèmes d'enregistrement audio. Il s'est agi d'annoter et de cataloguer l'ensemble des enregistrements disponibles des Opie, qui contient, entre autres, du matériel non publié dans les ouvrages des auteurs, et en particulier certains jeux extrêmes, scatologiques, mais aussi des jeux classiques et une partie des jeux influencés par les médias (qui sont sous-représentés dans les sources publiées). ${ }^{3}$

Comme l'indique le site de la British Library «The Opie Collection of Children's Games and Songs", la collection Opie des jeux et chansons d'enfants, est un ensemble d'enregistrements réalisés par Iona Opie entre 1969 and 1983 et déposés par celle-ci en 1998. La continuité est liée non seulement à la démarche mais aussi aux résultats, les jeux et chants d'aujourd'hui y compris dans leur relation forte aux médias étant dans une logique proche des sources présentées par Iona et Peter Opie.

4 Les différences sont à chercher dans la façon d'analyser les données, en s'éloignant des pratiques et théories du folklore traditionnel, cette spécialité relativement autonome à l'époque de l'ethnographie, au profit d'une approche au croisement d'une 
anthropologie contemporaine et de l'étude la culture médiatique des enfants, ellemême à la croisée de la sociologie de l'enfant et des media et cultural studies. Une autre différence est bien entendu le développement et l'apparition et nouveaux médias. A la radio et la télévision s'associent aujourd'hui les jeux vidéo et internet.

En effet au travail sur les données recueillies dans le passé s'ajoute un travail ethnographique de moins grande ampleur, compatible avec les modalités de financement de la recherche qui n'encouragent pas la fréquentation de mêmes lieux pendant 30 ans! Il s'agit d'une recherche dans deux écoles primaires, l'une située à Sheffield, l'autre à Londres, qui a permis de recueillir des données (cette fois sous forme d'enregistrements vidéo) concernant les jeux, comptines et chants des enfants en cour de récréation. C'est là où les questions des chercheurs différent et renvoient à celles qui peuvent se poser en ethnographie et sociologie de l'enfance. La question centrale est celle de l'agency des enfants, leur capacité à créer, à être des agents et non des récepteurs passifs. L'intérêt est ainsi porté sur les modifications apportées par chaque performance, chaque enfant. Il s'agit d'en trouver les sources mais aussi de mettre en évidence les inventions constantes, ou pour reprendre le concept de Corsaro ${ }^{4}$, auteur cité dans l'ouvrage mais dont cette notion n'est pas reprise, la «reproduction interprétative " propre à l'activité des enfants et qui me semble plus adaptée (ou plus facile à traduire en français) que celle proposée de cultural rehearsal, répétition (au sens théâtral) culturelle. Sont mis en lumière de façon centrale le contexte social, et la question du genre dans le jeu.

6 Mais les enfants interviennent également comme participants à la recherche ce qui rejoint le courant, particulièrement dynamique au Royaume Uni, qui implique les enfants dans les recherches les concernant. Deux éléments sont essentiels dans cette participation et documentés chacun par un chapitre de cet ouvrage collectif.

Il s'agit d'une part la création d'un site web où les enfants apparaissent comme codesigner. C'est à partir de leurs suggestions que celui-ci a été conçu mais nous retrouvons ici l'instabilité du net dans la mesure où ce site baptisé Playtime, conçu et hébergé par la British Library n'est plus accessible à l'adresse donnée dans le livre et semble avoir disparu du web ce qui interroge sur la logique de conservation de l'institution. De même un documentaire réalisé à l'occasion n'est pas consultable sur le net. On peut imaginer que des questions de droit à l'image ont pu rendre le processus difficile mais il est dommage d'avoir fait disparaître toute trace d'un site et de l'implication des enfants. Seuls les adultes à même de maîtriser l'écrit peuvent ainsi laisser des traces durables.

D'autre part a été conçu le Game Catcher (l'attrapeur de jeu pourrait-on traduire), un jeu vidéo destiné à permettre de saisir les mouvements des enfants lors des jeux et comptines. Il leur permet de jouer avec une interface combinant les principes de la Nintendo Wii et du Microsoft Kinect tout en pouvant être utilisée dans un but de recherche. Il s'agit d'une expérimentation, d'un projet exploratoire qui expose une manière de noter les mouvements d'un jeu de façon précise sans utiliser des instruments lourds de captation des mouvements (capture motion). En effet les concepteurs souhaitent que cela prenne la forme d'un jeu pour les enfants d'où tout un travail de test avec ceux-ci de façon à saisir dans quelle mesure le principe de fonctionnement est compris. La compétence des enfants en matière de jeux vidéo et de dispositifs techniques afférents leur permet effectivement d'utiliser sans problème le 
Game Catcher. Les chercheurs ont été attentifs à leur retour d'expérience pour percevoir leur intérêt pour un tel jeu ouvert (qui ne donne pas d'objectif précis).

Au-delà de ces diverses dimensions qui sont en relation étroite avec l'étude ethnographique (diffusion par le site web, recherche d'un nouvel outil de captation qui intègre au mieux les enfants comme participants, comparaison avec les études historiques des Opie) celle-ci est bien le centre de la recherche, et une présentation plus complète des résultats fait l'objet d'une autre publication' ${ }^{5}$.

10 L'étude ethnographique permet de retrouver le répertoire des Opie mais aussi de nouvelles modalités de jeu: en particulier des jeux liés à la culture médiatique des enfants, des jeux de faire semblant qui mélangent univers familial et monde fantaisiste (super héros, contes, sorcières et Zombies). Quatre chapitres de cet ouvrage sont consacrés à la présentation de certains résultats de cette étude.

11 Celui de Julia Bishop s'intéresse à la transmission online et offline d'un clapping game, expression qui n'a pas d'équivalent en français et que l'on pourrait traduire par comptines/jeux de main (tous les jeux de mains ne sont pas de comptines et réciproquement). Elle montre comment aujourd'hui s'articulent des transmissions locales (au sein de la cour de récréation) et globales, en particulier à travers les vidéos de clapping games que l'on trouve sur Youtube. Les enfants observés, témoignant ainsi de leur capacité d'agir, mélangent, mixent (remixent) des éléments issus de Youtube avec ceux qui circulent dans la cour de récréation. Les enfants ne sont pas seulement des joueurs, ce qu'on leur accorde volontiers mais aussi des concepteurs de leurs jeux et des médiateurs dans le sens où ils établissent des ponts entre diverses sources. En suivant dans la cour une "même» comptine-jeu de main, l'auteure montre les variations entre les performances des unes et des autres (ce jeu ne concerne que les filles), variations qui portent sur les trois dimensions de cette communication multimodale : les paroles, les mouvements du corps et la musique ou son rythme. Les performances peuvent révéler des logiques de transmission mais aussi le fait de mélanger de façon originale diverses sources. Tout cela se déroule dans le contexte social spécifique d'une cour de récréation avec des logiques relationnelles. Pour employer un autre vocabulaire que celui utilisé par les auteurs, on peut voir le rôle d'un script préexistant qui organise la performance, mais les variations dans les performances élargissent le répertoire proposé et permettent des modifications du script.

12 Le chapitre de Chris Richard pose la question de la place des jeux de bagarre, rappelant qu'ils font partie depuis bien longtemps (l'auteur remonte à son enfance) de la culture ludique enfantine, des traditions et qui sont récusées par les enseignants lors même que pour les enfants les jeux de bagarres diffèrent des bagarres même s'il existe entre les deux une frontière floue. D'où l'importance des régulations scolaires dans les cours à la différence d'espaces non contrôlés, comme la rue, mais qui sont aujourd'hui de moins en moins des espaces de jeu. La cour peut ainsi être investie de projets pédagogiques (coopérer, libérer son énergie, etc.), sans doute plus en Angleterre qu'en France, ce qui conduit à traquer les jeux de bagarres porteurs d'images très négatives pour les enseignants interrogés qui les associent à la violence voire au crime. D'où l'existence de jeux qui se développent quand les enseignants ne regardent pas mais qui sont présents dans la cour. On peut en particulier noter l'importance des jeux de poursuite et de bagarre entre genres auxquels les filles participent positivement (en 
allant provoquer les garçons pour produire ce jeu). Ce gender war est un jeu de chasse, de poursuite dont Corsaro (1997) évoque l'importance dans la culture enfantine.

Le chapitre de Jackie Marsh s'intéresse à l'articulation entre les relations en ligne et hors ligne, recherchant d'éventuelles connexions entre exclusion online et dans les jeux réels. Elle ne perçoit pas de relation, ce qui n'est pas étonnant, les deux univers de jeux formant des mondes séparés et spécifiques. Si l'enquête montre l'importance des relations en ligne, elle ne concerne pas pour autant tous les enfants, certains n'y ayant pas accès faute de matériel et d'autorisation parentale, mais cela n'a pas d'incidence sur les participations aux jeux offline, dans la cour de récréation, les réseaux d'amitiés online et offline pouvant être très différents.

14 Le chapitre de Rebekah Willett analyse le recyclage des cultures, en particulier médiatiques, dans le jeu, qu'il s'agisse de télévision, d'internet ou de jeux vidéo. C'est sous la figure du remix, caractéristique de la culture post-moderne, qu'est pensée cette relation qui peut prendre plusieurs formes:

15 - l'allusion onomastique ou utilisation du nom mais pour un jeu traditionnel (chat) qui ne change pas

16 - le syncrétisme, qui consiste à insérer des éléments médiatiques dans un jeu traditionnel, comme changer le jeu de chat en utilisant des éléments venant de Spiderman

17 - la mimésis ou imitation des éléments issus des médias qui donnent la structure même du jeu

18 - La parodie

19 D'autres propositions de catégorisation sont faites, montrant surtout la limite de l'exercice, relevant du jeu du chercheur mais éloigné de celui de l'enfant qui ne connaît pas de telles catégories et qui renvoie plutôt à un continuum où les différents éléments interfèrent. Il s'agit d'un processus d'hybridation, de "bricolage ludique ». Ce chapitre se termine par une étude de cas, un jeu complexe se référant à la télévision: des enfants jouent le rôle de personnes à l'écran et d'autres constituent le public. Ils peuvent ainsi changer de chaîne ce qui conduit à un changement de jeu, comme le passage d'une émission de chansons à une série avec des pirates. On peut voir ici une mise en abîme tout à fait exceptionnelle de la relation avec la télévision comme source du jeu, révélant l'importance de l'agency mais aussi de l'articulation des différentes initiatives des enfants pour produire une activité collective durable.

Comme le dit Willett (page 149) « la culture Remix dans la cour de récréation implique la sélection des ressources qui peuvent fonctionner dans le contexte très spécifique d'un jeu singulier ». Reprenant le concept foucaldien d'hétérotopie, elle montre que le jeu apparaît comme un lieu autre, imaginaire, parfois transgressif où se rencontrent monde virtuel et espace de la cour de récréation. 


\section{NOTES}

1. On peut consulter le rapport final de la recherche à l'adresse suivante: http:// projects.beyondtext.ac.uk/playgroundgames/uploads/end_of_project_report.pdf

2. Les principaux ouvrages sont : Iona Opie The People in the Playground, Oxford, Oxford University Press, 1993 ; Iona et Peter Opie, The Lore and Language of Schoolchildren, Oxford, Oxford University Press, 1959 ; Children's Games in Street and Playground, Oxford, Clarendon Press, 1969 ; The Singing Game. Oxford, Oxford University Press, 1985

3. On trouve ce matériel à l'adresse,

http://sounds.bl.uk/Accents-and-dialects/Opie-collection-of-children-s-games-and-songs-, celle donnée dans le livre ne fonctionnant pas et révélant ainsi les problèmes d'instabilité du net par rapport à l'écrit.

4. William Corsaro, Sociology of Childhood, Thousands Oaks (Ca), Pine Forge Press, 1997

5. Rebekah Willett, Chris Richards, Jackie Marsh, Andrew Burn et Julia C. Bishop, Children, Media and Playground Cultures. Ethnographic Studies of School Playtimes, Basingstoke, Palgrave Macmillan, 2013 